\title{
Follow-Up Study of Torabi et al. A Cross-Sectional Survey on the Inclusion of Tobacco Prevention/ Cessation, Nutrition/Diet, and Exercise Physiology/ Fitness Education in Medical School Curricula
}

\author{
Conner McKinney and Mohammad R Torabi* \\ Indiana University-Bloomington, School of Public Health, USA
}

*Corresponding author: Mohammad R Torabi, Indiana University-Bloomington, School of Public Health, USA.

Received Date: June 24, 2019

Published Date: July 24, 2019

Abstract

Currently in the United States chronic diseases are the leading cause of death and disability. Physicians of today possess the greatest opportunity to impact the long-term health of their patients through preventative medicine. Despite the transition from infectious diseases to chronic conditions, many physicians today are inadequately prepared to practice preventative medicine. In addition, medical schools are largely failing to adjust curriculum to best address this disease transition. The purpose of this study is to provide a follow up report of an article published by Mohammad R. Torabi, PhD; Ran Tao, MPH; Stephen J. Jay, MD; and Courtney Olcott, MS, through a literature review of the inclusion of tobacco prevention/ cessation, nutrition/diet, and exercise physiology/fitness education in medical school curricula from 2011 to present day [1]. The previous article found that the areas of tobacco prevention/cessation, nutrition/diet, and exercise physiology/fitness received the least amount of attention in medical school's curriculum despite many students feeling that these topics hold direct relevance to their learning, and offered recommendations in an attempt to address this gap in education. Results of the current study found that since 2011 there is little evidence of medical schools adopting the recommendations made by the previous article. The literature review also provided evidence that education pertaining to tobacco prevention/ cessation and nutrition/diet remains limited while education of exercise physiology/fitness has shown a slight increase. Results of this study indicate that gaps in medical school curriculum are still prevalent, and further research should assess the prevalence of education of these topic areas and the strategies medical schools are utilizing to best prepare students to practice preventative medicine to best address chronic diseases today.

\section{Introduction}

In the United States and around the world disease trends have dramatically shifted away from communicable diseases of the past to more predominantly prevalent noncommunicable chronic diseases. According to the World Health Organization [2], major chronic diseases such as cardiovascular diseases (CVD), cancer, chronic obstructive pulmonary disease (COPD), and type two diabetes currently account for $60 \%$ of all deaths and $43 \%$ of the global burden of disease. One of the most alarming aspects of this disease trend is found in the scope of who is affected. Unlike typical diseases of the past that were typically confined a handful of demographics or countries, chronic disease [3] "rates are accelerating globally, advancing across every region and pervading all socioeconomic classes" (1). Current CDC research indicates that this trend is just as prevalent in the U.S. as it is around the world.
Current leading causes of death and disability in the U.S. are related to heart disease, cancer, and diabetes (2). According to the CDC on average 6 out of every 10 adults in the U.S. have a chronic disease, and 4 out of every 10 adults have two or more chronic diseases (3). In an attempt to address this worldwide health concern, the health care community has begun to transition its attention to more preventative measures. While many of these diseases can be prevented by focusing early on to patients specific biological and behavioral risk factors, it is imperative for future efforts to focus on the leading causes of these diseases such as high blood pressure, poor nutrition, tobacco use, and physical inactivity.

An earlier study exploring the inclusion of tobacco prevention/ cessation, nutrition/diet, and exercise physiology/fitness education in medical school curricula by Mohammad R. Torabi, PhD; Ran Tao, 
MPH; Stephen J. Jay, MD; Courtney Olcott, MS determined that of the three topic areas exercise physiology/fitness education received the least amount of attention when looking at areas such as participant impression of relevance, level of training, and level of students ability to give patients advice on the topic. Furthermore, the study reported that even though medical schools and medical students view tobacco prevention/cessation and nutrition/diet education relevant or extremally relevant, both areas "are not covered thoroughly in medical school curricula, and that there is a demand by medical school students and faculty to increase education in these areas" (4). Notably, these three educational topics are directly relevant to the growing chronic disease issues in both the United States and around the world.

In an attempt to fill in gaps found in the literature at the time, the study recommended a possible revision of medical school curricula and how that information is delivered to students. One possible solution highlighted by the research involves a transition from the typical lecture delivery of information to more applied education strategies such as case studies, patient-centered counseling, critiqued interactions, and clinical labs. The study goes on to indicate that these alternative methods of education have been shown to provide positive results in areas of tobacco prevention/cessation, nutrition/diet, and exercise physiology/ fitness and should be considered when addressing these issues later on. The purpose of this current study is to provide a followup report through a literature review of the inclusion of tobacco prevention/cessation, nutrition/diet, and exercise physiology/ fitness education in medical school curricula from 2011 to present day.

\section{Literature Review}

When addressing the growing issues caused by chronic diseases in the United States, educational topics such as tobacco prevention/ cessation, nutrition/diet, and exercise physiology/ fitness are essential components for medical students to understand. Revision in medical school curricula could provide a positive impact on preventative practices as patients today are accessing healthcare through a variety of providers and more than ever should be receiving a consistent message from all of their encounters. Furthermore, students in differing health professions need to have more of an understanding of the roles played by other disciplines in order to best address preventative approaches to chronic diseases Zenzano et al. [4].

\section{Tobacco prevention/Cessation}

Currently in the United States, the CDC is reporting that more that 16 million Americans are living with a smoke-related disease. While rates of smoking have started to slowly decline since 2005, $14 \%$ of the population as of 2017 reported that they were still current smokers. The findings of the previous article by Torabi et al. indicated that of the medical schools surveyed only $39.29 \%$ felt that their students were very knowledgeable in the area of tobacco prevention/cessation. In addition, of those medical schools surveyed only $28.57 \%$ believed that their students were well trained in tobacco prevention (5). Further research into the literature available today does not suggest that drastic improvements have been made to medical school's curricula on tobacco prevention/ cessation.

In the United States the most widely supported physiciandelivered intervention to help patients quit smoking involves a strategy known as the 5A's. Physicians are advised to Ask their patients about their current use of tobacco products, Advise current smokers clearly to quit, Assess whether or not the patient is ready to quit, Assist patients willing to quit (by referring to a cessation program, or exploring possible medication options), and Arrange a follow up preferably within the first week the patient begins to quit (6). The usage of this intervention was looked at by Rashelle B. Hayes et al. in their article looking to develop and implement a comprehensive medical school curriculum for teaching the effective delivery of tobacco treatment counseling and to evaluate its impact on acquired student tobacco treatment and counseling skills within the context of a group randomized control trial. Their article states that "between 32 and $54 \%$ of primary care physicians did not implement any 'Assist' behaviors with patients...and about 77\% had not 'Arranged' for follow-up care" Hayes et al. [5]. A possible explanation behind the poor understanding and implementation of the 5A's explored by this article could be due to the fact that there is not one standardized curriculum for medical school students on the topic of tobacco prevention/cessation. Medical school curricula varies among schools and from year to year within schools. As a result, medical students continue to receive inadequate and inconsistent training in tobacco prevention/cessation efforts. Further research should address training standardization in medical schools on this topic Hayes et al. [5].

The findings from the study conducted by Hayes et al. [5] are further supported by the article published by Judith K. Ockene et al. [6] that looked to assess the effects of a multi-modal tobacco dependence treatment curriculum on medical students' counseling skills. This article points out the important role that physicians play in helping their patients to quit smoking. As in the Hayes article, Ockene et al. [6]. highlights that despite evidence of the positive impact the $5 \mathrm{~A}$ intervention can have, "the use of the $5 \mathrm{~A}^{\prime}$ s among physicians is limited as is the amount of tobacco treatment training that physicians receive" Ockene et al. [6]. In an attempt to address this issue, the study developed, implemented, and evaluated a comprehensive multi-modal education (MME) intervention for teaching effective delivery of tobacco dependence treatment to medical students as opposed to traditional education practices. While the results of the study did not provide statistically significant differences between those who received the MME and traditional education, MME students did complete more tobacco counseling behaviors on their objective structured clinical examination checklist, and were statistically more likely to complete individual "Assist" and "Arrange" items from the 5A's than those students who received traditional education. Further research into statistically 
significant education interventions for medical schools' inclusion and delivery of tobacco prevention/cessation curricula should be addressed Ockene et al. [6].

One of the most challenging aspects of preventative medicine is the lack of an evaluation system to better understand the scope of preventative practices being taught and how well that information is being implemented. In an attempt to address this challenge Kathleen M. Mazor et al. [7] designed a study to implement an assessment of medical students' tobacco dependence treatment skills with hopes that this model can be used by other educators providing them with a highly standardized assessment across multiple medical schools. Physicians hold the biggest opportunity to impact rates of smoking in the United States, with nearly $70 \%$ of current smokers having at least one physician's appointments a year it is imperative for providers to have adequate training in tobacco prevention/cessation Mazor et al. [7]. This study designed and implemented an assessment involving ten medical schools and used a 33-item checklist with detailed criteria and examples to score students performances during an objective structured clinical examination. Results of this studies assessment of the ten medical schools involved revealed that on average students performed only 8 of the 33 items on the checklist, and that for 16 of the 33 behaviors only $10 \%$ of students performed those behaviors. Furthermore, this study discovered that medical students tended to focus more on pharmacotherapy as opposed to behavioral strategies such as arranging for referral support or discussing barriers to be quitting Mazor et al. [7]. While this study was able to develop and implement an assessment of medical students' understanding of tobacco dependent treatment, the results indicate that current medical school curricula fall short of providing students with the knowledge they need adequately assist patients.

\section{Nutrition/Diet}

Nutrition and healthy body weight is one area that Healthy People 2020 is attempting to make an impact. The goal of Healthy People 2020 is "to promote health and reduce chronic disease risk through the consumption of healthful diets and achievement and maintenance of healthy body weights" (10). To highlight how important this issue is, Healthy People 2020 [8] indicates that a healthy diet is not only beneficial for weight management but can also reduce risks for many chronic diseases including heart disease, high blood pressure, type 2 diabetes, and some cancers (10). The World Health Organization [9] also cites an unhealthy diet as one of the leading global risks to health (11). With all of the different aspects of health that nutrition and diet can impact it should be a topic of importance when it comes to medical school. Findings from the previous article by Torabi et al. however indicated that of the medical schools surveyed only $17.86 \%$ felt that their students were very knowledgeable on the topic of nutrition/diet, and only $21.43 \%$ felt that their students were well trained in the area of nutrition/ diet (4). Results from a further look into available literature today does not suggest positive improvements in the competencies around nutrition/diet.
In the United States approximately $39.8 \%$ of the adult population and $18.5 \%$ of all children and adolescents are obese (12). Currently fewer than 1 in 10 children and adults regularly consume the daily recommended amount of vegetables, and only 4 in 10 children and 1 in 7 adults are getting the daily amount of fruits (13). While research indicates that individuals who are at, and able to maintain, a healthy weight [10] are less likely to develop chronic disease risk factors, it is important to understand what is being done in medical schools to promote better nutrition [11] in an effort to prevent future chronic disease issues (10). The article from Kelly M. Adams et al. looked to assess the current state of nutritional education in U.S. medical schools and to compare it with the recommended instructional targets. Highlighted in the article is the fact that physicians have the potential to drastically impact the nutritional habits of their patients given that physicians are the ones who typically see patients when they could use nutritional aid the most. Despite the opportunity's physicians have to positively impact their patients' health status in regard to nutrition and diet, many lacks the knowledge needed to accurately detect symptoms of nutritional problems or what to do if they do notice. This gap in education could partially be related to the fact that "The Association of American Medical Colleges has recently declined to incorporate nutrition into their new blueprint for medical competencies" Adams et al. [12]. Upon completion of their assessment of the 121 participating medical schools through an online survey, this study reported that 86 of the 121 schools fail to meet the recommendation of a minimum of 25 hours of nutrition education. In addition, 12 institutions reported no nutritional education. The lack of consistent nutritional education around the United States means that only $24.6 \%$ of all medical students receive adequate instruction on the topic as highlighted by the article Adams et al. [12]. With poor nutrition being a direct contributor to chronic disease issues in the United States and around the world further research should explore ways to increase consistently proper nutritional education across all medical schools.

Another article looking to describe the educational experiences, attitudes, and practices related to nutrition among cardiovascular professionals supports the idea presented by Adams et al. that physicians today are not receiving adequate education related to nutrition. The article by Stephen Devries et al. utilized an online cross-section survey designed to specifically assess cardiovascular specialists nutritional education and practice related to nutritional counseling. With poor diet being directly related to health issues such as high blood pressure and heart disease, and one of the main causes of morbidity and mortality in the United States, it is easy to see why an understanding of cardiologist's nutrition education is needed Deveris et al. [13]. Results from the survey indicated that $31 \%$ of cardiologists and $21 \%$ of fellows-in-training did not recall receiving nutritional education in medical school, $59 \%$ of cardiologists did not recall receiving nutritional lectures during their internal medicine residency, 90\% of cardiologists reported no or minimal nutrition education during fellowship training, and $56 \%$ reported that following their fellowship training they received 
no nutritional education; while only $20 \%$ did report receiving two or more hours of nutritional education per year. Interestingly the article points out that this lack of education does not stem from a lack of interest by cardiologists since 53\% reported that they would like to partake in an online educational series discussing nutrition and lifestyle Devries et al. [13]. This report adds to the literature supporting the idea that nutritional education in medical schools is inadequately preparing physicians to handle growing nutritional/ diet issues facing the country and simultaneously highlights areas where possible improvements could be implemented.

While it has become clear that there are gaps in nutritional education, more efforts to address this issue and propose possible solutions are needed. An article by Lazlo N. Kiraly et al. [14]. aimed not only to address the problems facing nutrition education of physicians but also reviews alternative education models to traditional nutritional fellowships and looks at other examples of adult learning that could be used in the nutritional education of physicians. Traditionally medical school education is separated into two two-year portions. The first two years are typically pre-clinical didactics which is where the majority of nutritional education takes place if the medical school has chosen to include it. The second two years are typically clinical experiences. This article points out that by not incorporating the nutritional component alongside the clinical experiences, the result is a lack of nutritional application to real patients. This lack of experience is then only perpetuated by the fact that there are fewer and fewer physician nutritional specialists or leaders in residency. For this reason, Lazlo et al. [14] recommends that traditional nutritional fellowships and simply increasing lecture time of nutritional information will not positively impact the state of nutritional education in the United States. A possible solution to this issue that requires further research would be the implementation of short immersion courses that could incorporate close mentor ships, and adult learning techniques including clinical experiences, literature reviews, and multi-disciplinary interactions Kiraly et al. [14]. In order to best address the issue of nutritional education in medical school's further research into alternative teaching methods and curricula development are required.

\section{Exercise physiology/Fitness}

Set back in 2008 the U.S. Department for Health and Human Services [15] released the first ever Physical Activity Guidelines for Americans. These guidelines were then adopted by the Healthy People 2020 initiatives citing strong scientific evidence supporting the benefits that regular physical activity can have on both adults and children. The Healthy People 2020 outline explains that physical activity and exercise are not just for teens and young adults [16]. The benefits of including even just minimal amounts of physical activity can increase health and quality of life for all Americans; including lowing the risk for chronic diseases such as coronary heart disease, high blood pressure, type 2 diabetes, breast and colon cancers, as well as depression (17). Despite the evidence supporting the benefits physical activity can have on our population, the CDC currently reports that only half of Americans receive adequate amounts of physical activity needed to reduce chronic disease risks. In addition, the result of such a lack of exercise costs the United States $\$ 117$ billion in health care costs (18). Results from the Torabi et al. report indicated that of the medical schools surveyed $10.71 \%$ felt that their students were very knowledgeable on the topic of exercise physiology/fitness, and that only $3.57 \%$ felt their students were well trained in this area (4). Similarly, to the outcomes of tobacco prevention/cessation and nutrition/diet the literature review does not indicate positive changes in curricula in medical schools.

An article out of the University of Oregon looked to provide an update on the current state of U.S. medical schools education curricula for physical activity related content. The article starts out by addressing the fact that since the reform brought about by the Flexner report back in 1910 there has not been any drastic changes made to medical school curriculum. Physicians of today are still traditionally treating diseases as opposed to working on more preventative measures which is remnant of the changes brought about by the Flexner report and may not be best suited for the chronic diseases our nation faces today. This article reviewed all of the accredited MD and OD institutions in the United States and Canada by accessing their publicly available websites. Findings of this articles review indicated that of the 118 institutions whose curriculum information was available $51.7 \%$ did not offer a single course in this area and $66.9 \%$ did not offer elective courses addressing physical activity related content Park [17]. Of the schools that did offer coursework regarding physical activity related content, courses were typically related to sports medicine at $45 \%$ or exercise physiology at $40.9 \%$. Course work related to preventative medicine addressing this area was only found in $8.1 \%$ of medical schools, and course work pertaining to lifestyle medicine and behavioral counseling were only reported in $4.7 \%$ and $1.4 \%$ respectively Park [17]. These results highlight the fact that the vast majority of medical students are inadequacy prepared to assist patients with regards to physical activity and fitness related issues and lack the behavioral counseling skills needed to help them make changes to positively impact their overall health.

In an effort to add to the available literature on the topic of physical activity related curriculum in medical schools Mark Stoutenberg et. al [18] engaged U.S. medical school program directors in an attempt to determine the level and timing of physical activity training being provided in medical schools. This article recognizes the fact that by participating in some form of regular physical activity individuals have the chance to reduce their risk of many chronic diseases. Despite the scientific evidence behind this claim, "over the past two decades levels physical inactivity has continued to rise in the US" Stoutenberg et al. [18]. Citing that influences and advice from medical professionals has the best potential to change habits among patients, this article designed structured interviews in order to determine the level of training medical students are receiving relating to physical activity related content. Results of this study indicate that the inclusion of physical activity related courses are on the rise, but still have room for improvement. Of the 171 accredited medical institutions only 74 completed the 
structured interviews. From those schools 58 reported having some sort of physical activity and 31 programs reported that they felt as though the amount of physical activity related content was adequately preparing their students to counsel their patients in the future Stoutenberg et al. [18]. One possible limitation of this study could be the low response rate to the structured interviews. While this study does indicate that improvements are being made, future research should look to incorporate more institutions in order to provide better generalizability.

Another article looking to provide an update on the current status of the amount and type of physical activity education being offered in medical schools in the United States provides similar results to the Stoutenberg et al. [18] article. The article by Cardinal et al. applied content analysis methodology in an attempt to assess available M.D. and O.D. institutions website available coursework in the area of physical activity [19] education. The article starts out by addressing the science behind how regular physical activity is capable of providing "widespread preventative, restorative, and curative powers with value superior to any other individual lifestyle intervention or treatment, and in some cases, similar to available drug therapies used in preventing coronary heart disease, diabetes, rehabilitation following a stroke, and threatening heart failure" Cardinal et al. [20]. Results from this studies assessment show that in terms of prevalence of physical activity related education, of the 118 respondence 61 offered no courses related to the topic and 25 only offered one course. Similarly, to the Stoutenberg et al. [18] article, results from this study showed that when courses were offered, they were either in the area of sports medicine with 67 institutions reporting or in exercise physiology with 61 institutions reporting Cardinal et al. [20]. This study adds to the available literature and attempts to raise areas of concern regarding gaps in medical school curricula.

\section{Conclusion}

Upon completion of the literature review of available information on these three topic areas, it has become evident that major gaps are still present in medical school curricula in the United States. While rates of exercise physiology were seen to be on the rise, areas such as tobacco prevention/cessation and nutrition/ diet education remains limited and uncomprehensive. There was evidence of studies using methods suggested by the precious article by Torabi et al., such as patient-centered counseling and clinical application, however there is a lack of information providing evidence that these methods have been adopted or implemented by medical schools though out the United States. The current follows up study conducted a literature review of available articles pertaining to the three topic areas outlined, and current national prevalence information from various governmental sources. One possible limitation of this study would be the fact that medical schools were not contacted directly to assess whether or not they have implemented strategies recommended by the previous article. Future research should further assess the prevalence of education of these topic areas and the strategies medical schools are utilizing to best prepare their students to adequately counsel patients and implement preventative medicine to address the chronic diseases affecting our nation.

\section{Acknowledgments}

None.

\section{Conflict of Interest}

No conflict of interest.

\section{References}

1. Torabi MR, Tao R, Jay SJ, Olcott C (2011) A cross-sectional survey on the inclusion of tobacco prevention/cessation, nutrition/diet, and exercise physiology/fitness education inmedical school curricula. J Natl Med Assoc 103(5): 400-406.

2. (2019) Chronic Disease and Health Promotion. World Health Organization, USA.

3. (2019) National Center for Chronic Disease Prevention and Health Promotion, USA.

4. Zenzano T, Allan JD, Bigley MB, Bushardt RL, Garr DR, et al. (2011) The roles of healthcare professionals in implementing clinical prevention and population health. Am J Prev Med 40(2): 261-267.

5. Hayes RB, Geller A, Churchill L, Jolicoeur D, Murray DM, et al. (2014) Teaching tobacco dependence treatment and counseling skills during medical school: rationale and design of the Medical Students helping patients Quit tobacco (MSQuit) group randomized controlled trial. Contemp Clin Trials 37(2): 284-293.

6. Ockene JK, Hayes RB, Churchill LC, Crawford SL, Jolicoeur DG, et al. (2016) Teaching medical students to help patients quit smoking: outcomes of a 10-school randomized controlled trial. Journal of general internal medicine 31(2): 172-181.

7. Mazor KM, Jolicoeur D, Hayes RB, Geller AC, Churchill L, et al. (2015) Assessing medical students' tobacco dependence treatment skills using a detailed behavioral checklist. Teach Learn Med 27(3): 292-298.

8. (2019) Office of Disease Prevention and Health Promotion. Healthy People 2020 Physical Activity, USA.

9. (2019) World Health Organization. Healthy Diet, USA.

10. (2019) Centers for Disease Control and Prevention. Overweight and Obesity, USA.

11. (2019) Centers for Disease Control and Prevention. Nutrition, USA.

12. Adams KM, Butsch WS, Kohlmeier M (2015) The state of nutrition education at US medical schools. Journal of Biomedical Education.

13. Devries S, Agatston A, Aggarwal M, Aspry KE, Esselstyn CB, et al. (2017) A deficiency of nutrition education and practice in cardiology. Am J Med 130(11): 1298-1305.

14. Kiraly LN, Mc Clave SA, Neel D, Evans DC, Martindale RG, et al. (2014) Physician nutrition education. Nutr Clin Pract 29(3): 332-337.

15. (2019) US Department of Health and Human Services. 5 Major Septs to Intervention (the 5 A's), USA.

16. (2019) Office of Disease prevention and Health Promotion. Healthy People 2020, USA.

17. Park EA (2014) National Review of US Medical Education Curricula for Physical Activity-Related Content.

18. Stoutenberg M, Stasi S, Stamatakis E, Danek D, Dufour T, et al. (2015) Physical activity training in US medical schools: Preparing future physicians to engage in primary prevention. Phys Sportsmed 43(4): 388-394.

19. (2019) Centers for Disease Control and Prevention. Physical Activity, USA.

20. Cardinal BJ, Park EA, Kim M, Cardinal MK (2015) If exercise is medicine, where is exercise in medicine? Review of US medical education curricula for physical activity-related content. J Phys Act Health 12(9): 1336-1343. 